\title{
COVID 19 Emergency Multimedia Rehabilitation Assistance: Case Report
}

\author{
Gentile $\mathrm{G}^{1 *}$, Rizzano $\mathrm{V}^{2}$, Coppola $\mathrm{MT}^{3}$, Gargano $\mathrm{A}^{3}$, Scoppa $\mathrm{G}^{4}$ and Pagano $\mathrm{MC}^{5}$ \\ ${ }^{1}$ Director of the Department of Rehabilitation and Prosthetics, ASL Salerno, Italy \\ ${ }^{2} A B A$ Department Coordinator - Rehabilitation Center "Scoppa Foundation", ASL Salerno, Italy \\ ${ }^{3} A B A$ Therapist - Rehabilitation Center "Scoppa Foundation", ASL Salerno, Italy \\ ${ }^{4}$ Psychologist - Behavior Analyst - Rehabilitation Center "Scoppa Foundation", ASL Salerno, Italy \\ ${ }^{5}$ Faculty of Medicine and Surgery, University of Magna Graecia of Catanzaro, Italy
}

*Corresponding author: Gentile G, Director of the Department of Rehabilitation and Prosthetics, ASL Salerno, Italy

To Cite This Article: Gentile G, Rizzano V, Coppola MT, Gargano A, Scoppa G, Pagano MC, COVID 19 Emergency Multimedia Rehabilitation Assistance: Case Report. Am J Biomed Sci \& Res. 2021 - 11(4). AJBSR.MS.ID.001651. DOI: 10.34297/AJBSR.2021.11.001651.

Received: 眥 December 14, 2020; Published: 海 January 12, 2021

Keywords: Rehabilitation; Tele-assistance; Quarantine from Coronavirus 2019-nCOV

\section{Introduction}

The data concerning the Coronavirus 2019-nCov identify minors with complex chronic pathologies a population at increased risk and therefore neuromotor disability represents a target in need of particular attention. And in fact, ministerial and regional directives have recommended to family members and caregivers of fragile children to engage in behavior aimed at reducing the risk of contagion, including, among other things, the reduction of access to health and rehabilitation services for elective services. not urgent or postponed. Although children are less affected and have a lower risk of developing complications related to viral infection, there is actually no evidence that they, more difficult to limit in contacts, cannot act as vectors, in particular asymptomatic; and also, an increase in the frequency of children with Kawasaki disease has been reported, especially in the areas most affected by the epidemic.

\section{Contest}

The health situation relating to the Coid 19 emergency resulted in the suspension of the deferred Individual Rehabilitation Projects (PRI) aimed at children with disabilities, and this by virtue of the need to act social containment measures to limit the spread of SARS-CoV-2. It emerged for the children, for whom, suddenly, the rehabilitation treatments and the loss of contact with the reference operators took place, and for the caregivers, projected into an increased difficulty due to the lack of the usual competent support, it emerged the need to reorganize specialist rehabilitation services in order to guarantee continuity of care and assistance, ensuring the highest level of care compatible with the needs of public health and safety of care [1,2]. Significant attention, in this context, was paid to the conditions for which isolation in the home, the interruption of the relationship with the reference operators and the limitations on movements could determine greater clinical effects: children with autism or with ADHD, adolescents with severe psychiatric disorder, minors with neuromotor or motor deficits, especially if they have travel aids, children at socio-family risk. Pending the resumption of specialist services, children with autism spectrum disorder and their families were deemed in need of a structured facility aimed at maintaining the relationship with the affectively significant figures, represented, in particular, by the team of rehabilitation technicians of reference, considering, in this context, technology as a valid tool to make continuity of the rehabilitation process possible and competent support for families.

\section{Methodology}

The Multimedia Rehabilitation Assistance Project (ARM) was addressed to n. 16 children, 12 males and 4 females, aged 4-14 years, suffering from Autism spectrum disorder, treated with ABA 
(Applied Behavior Analysis) Methodology at the Rehabilitation Center Accredited with SSR, users, on average, of 10-18 weekly hours of therapy, divided between Center, school and home, to which, due to the aforementioned health emergency, the PRI was suddenly interrupted. The project received 6 children, 4 boys and 2 girls, aged 6-8 years (37\%). Membership was influenced, in particular, by two determinants: the condition of socio-environmental risk and the duration of the rehabilitation treatment, showing a correlation between adherence to the project, absence of social risk indicators in the family unit and duration of the user relationship -familycenter $\geq 18$ months, in relation to the affective and affiliation bonds established $[3,4]$. The proposed ARM was characterized by the possibility of parent-therapist contact of the ABA team in charge of the child, via WhatsApp and via Skype, for 24 hours / day and 7 days a week, at the onset of problematic behaviors of the child or difficulties experienced by the caregiver; on the support of the therapist, who indicated the appropriate procedures for the management of the problem to the caregiver who, in turn, put the techniques indicated in video-field; on parental training activities, delivered by the psychologist of the rehabilitation team through biweekly video calls, at the time and day chosen by the parents, which ensured the monitoring of users and the family routine. Among the procedures suggested to the caregiver to manage the child's problem behaviors, decreasing the frequency and at the same time reducing parental stress, the use of the Token economy techniques, education contract, daily agenda, differential reinforcement, toilet training was greater, reinforcing conditioning, educational control, task analysis, functional analysis, education programs, prompting, time out, videomodeling/ modeling, proactive and reactive strategies, symbolic game activity. These techniques, provided by the caregiver to the child, were carried out in video assistance and constantly monitored by the therapist until the behavior-problem was resolved.

The activities and services provided by the remote therapist were also found to be:

i. Counseling: indications in case the family had to manage one or more problematic behaviors deriving specifically from the lockdown situation.

ii. Support: definition of the daily diary

iii. Comfort: listening to the caregiver in times of difficulty; empathy towards the reported situation;

iv. Monitoring: observation-control of children's progress and skills acquired in therapy;

v. Parental training: monitoring of family routine and small users.

In the first 11 days from the suspension of the PRI, before the start of the teleassistance activity, in the baseline phase (A), and subsequently in the first 11 days of teleassistance, in the intervention phase (B), the following items were monitored:

vi. Educational control problems: Difficulty in sitting, difficulty in transitioning places and / or activities, difficulty in coming when called, difficulty in returning the reinforcer, difficulty in waiting, difficulty in executing simple or complex instructions.

vii. Problem behavior: Self-directed or heterodirect harmful behavior, throwing objects, banging your head, biting, screaming, crying.

viii. Sphincter control problems: Nocturnal or diurnal enuresis.

ix. Problems with desk activities: Refusal to carry out didactic or predidactic activities, refusal or difficulty in paying attention to the activities to be carried out, refusal or difficulty in completing an activity, difficulty concentrating, difficulty in scanning.

x. Problems in the natural environment: Difficulty in sharing games or activities, difficulty in autonomy, difficulty in structuring game times.

xi. Feeding problems: Difficulty in using cutlery, chewing, food selectivity.

xii. Parental stress: Assessment on a self-anchoring scale from 1 to 10 of the stress recorded by the caregiver. By requesting the caregiver to compile a daily list in which the number of episodes-problems emitted by their child were recorded and quantified, indicating the degree of parental stress, the daily number of individual behaviors was measured as a dependent variable of the project problem of the child in phase A and in B, the latter representing the independent variable of the project.

\section{Results}

Graph 1 shows, in the first 3-4 days of quarantine, that the daily number of problem behaviors increases not exponentially, suggesting that, in the first phase of suspension of the PRI, a maintenance of the skills acquired is achieved, presumably for remembrance effect of the treatments received, while, from the 4 th to the 11th quarantine day, an exponential increase in the daily number of behavior-problems is appreciated (+ 20\% / day). The problem areas emerging after the first 4-5 days of interruption of the PRI concern the items of educational control, problem behavior and desk activities, while the areas with a later onset, emerging after 5-6 days of suspension of therapy, concern the sphincter control problems, feeding and problems in the natural environment. Parental stress appears from the beginning of the suspension of the PRI, remaining high for the entire period $A$. The graph also highlights that, in the first 3-4 days of remote assistance, 
in intervention phase B, positive results are recorded in the items relating to problems the start of remote assistance, there are problems with educational control, desk activities and problem behaviors. Parental stress is already reduced in the 3rd day from the beginning of teleassistance, in fact in connection with the first clinical improvements verified for the child. From the comparison of the data collected at the beginning and end of the baseline and at the end of the intervention, in table 2 , the overall effectiveness of the remote assistance treatment is appreciated for all the target children of the intervention. In all the areas explored, in fact, there was a significant daily increase in the number of problemepisodes resulting from the interruption of the PRI and a significant reduction in the number of episodes, in relation to the remote assistance provided. The best results were recorded in the area of educational control, where, at the end of the intervention, each child has the same number of daily quarantine episodes. Parental stress recorded an average value per individual caregiver of 7.5 on a scale from 1 to 10 already on the first day of suspension of the PRI, reaching the value of $10 / 10$ at the end of the suspension phase; comparatively, the reduction of parental stress is already present in the initial stages of remote assistance, in correlation with the clinical improvements presented by the child, and reaches, at the end of the intervention, values much lower than the value recorded at the beginning of the suspension of the treatments [5-7].

\section{BASELINE (A)}

\section{INTERVENTO (B)}

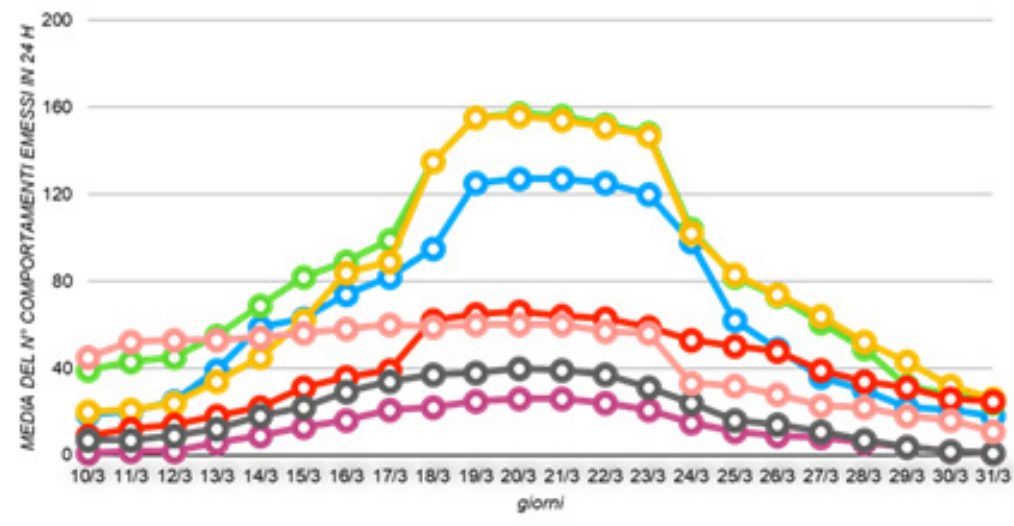

giomi

Graph 1: Trend of episodes-problem in baseline phase A and in intervention phase B.

\section{Conclusion}

Children with disabilities, and their families, represent a target that cannot guarantee the management of social and health risks during the COVID-19 emergency; in fact, for this population, the stress linked to the pandemic and the related containment measures adopted have a negative impact on the health of individuals and the family. In this scenario, the protection of competent resources, responsible for taking care of and managing assistance, has been a priority for the organization of local services. Therefore, considering that the health level of children with disabilities affects, specifically, the clinical, socio-family and rehabilitation aspects, given the need to guarantee them the maximum functional recovery and the maintenance of the skills present, have been identified, through specific experiential and professional approach, new operational scenarios aimed at ensuring continuity of rehabilitation services for children with autism spectrum disorder, evaluating, in this area of intervention, ARM as an innovative methodology. This case report, characterized by the originality of the proposed intervention which is not currently reflected in the common clinical practice of the health condition described, provides useful indications regarding the reorganization of rehabilitation services in the developmental age; in particular, enhances the contribution of the ARM as a care opportunity, recognizing the validity of the therapist's previous work on the skills acquired by the child, which has facilitated the generalization through the parental figures in the home, for the effectiveness of the results, and on the skills of the caregiver, in the unprecedented emergency situation of the therapist's arm and mind, these conditions which, by favoring the implementation of the suggestions and the correct execution of the remote procedures, have allowed to overcome the criticality of the lack of direct contact with the children, essential situation in the work of the therapist.

\section{Take a Message}

Considering, in the aforementioned epidemiological situation, the remodulation of health services as an absolute necessity, capitalizing on the experiences recorded today can represent the added value of our health system. An organic review of the care, 
support and care pathways for the disability that provides for the possibility of continuous socio-health contact with children and families through the ARM, can identify this method, initially born pending the recovery of rehabilitation services and effectively provided by guaranteeing high level of personal and collective safety in compliance with the measures to contain the health emergency from COVID-19, as a system resource. The relevance of the impact that the results obtainable from an ARM program, implemented to integrate codified rehabilitation settings, can determine in current clinical practice, combined with its sustainability, open up new possibilities for children, families and operators. In terms of disability, ARM, as an integrative tool of rehabilitation activity, can, in fact, be usefully included in the PRI of the child and family; it must necessarily be proactive, be able to make users perceive that the treating team is present as always but with new modalities, be able to manage any initial regressions of the skills acquired by the child, as well as able to verify the results of the online activity within the systemic monitoring of PRI outcomes. More generally, remote activity, structured within specific healthcare paths for defined targets, both as a temporary tool in emergency or shortterm situations, and as a useful methodology for clinical monitoring or transition of care, can significantly contribute the overall improvement of the quality, efficiency and sustainability of pediatric services. In all its fields of application, the ARM must be guaranteed to all children, avoiding their selective placement on more favorable socio-family situations, facilitating maximum involvement of the family, and of the caregiver in particular, overcoming situations of social distress -environmental, and this guarantees equity and equal opportunity to access services. And this, in order to rethink care, given the strength of the new perspective, planning each take charge of the child, and the fragile child, maintained, translated and integrated by the proposed innovation, with priority to situations of greater complexity, as a neighbor horizon of modern pediatrics.

\section{References}

1. www.regione.campania.it

2. www.assotaba.it

3. www.sip.it

4. https://www.abiliaproteggere.net

5. https://www.sidin.info/home

6. www.regione.campania.it

7. http://www.salute.gov.it/portale/temi 